CIC. Cuadernos de Información y Comunicación ISSN: 1135-7991

http://dx.doi.org/10.5209/ciyc.64638

\title{
Sistemas regulatorios de la publicidad institucional y su eficacia: la Junta de Comunidades de Castilla-La Mancha como anunciante
}

\author{
Jesús de la Cruz de Julián"; Ana María López Cepeda²; Belén Galletero Campos ${ }^{3}$
}

Recibido: 25 de abril de 2019 / Aceptado: 30 de mayo de 2019

Resumen. La publicidad de las administraciones públicas es una fuente más de ingresos para los medios de comunicación. Sin embargo, al tratarse de fondos de carácter público, este tipo de campañas ha precisado una reglamentación específica. Actualmente hay once comunidades autónomas con legislación en la materia y una norma de carácter nacional. Castilla-La Mancha es una de las seis autonomías que carece de ley regional, pero desde 2016 la contratación de las campañas de la Junta de Comunidades se rige mediante Acuerdo Marco. El presente trabajo, centrado en la publicidad institucional de la Junta de Castilla-La Mancha durante los años 2016, 2017 y 2018, tiene como objetivo comprobar si este nuevo sistema regulatorio ha mejorado la transparencia y la objetividad en los criterios de reparto. Se examinan las inserciones publicitarias del Gobierno autonómico (2016-2018) y se comparan con las métricas habituales de medición de audiencias.

Palabras clave: Publicidad institucional, legislación, Castilla-La Mancha, soportes, sectores.

\section{[en] Regulatory systems for institutional advertising and their effectiveness: the Junta de Comunidades de Castilla-La Mancha as advertiser}

\begin{abstract}
Advertising from public administrations is an income for the media. Public administration advertising is another source of revenue for the media. However, since they are public funds, this type of campaigns has required specific regulations. There are currently eleven autonomous communities with legislation on the subject and one national standard. Castilla-La Mancha is one of the six autonomous regions that lacks a regional law, but since 2016 the recruitment of the campaigns of the Junta de Comunidades is governed by Framework Agreement. This work, focused on the institutional publicity of the Junta de Castilla-La Mancha during the years 2016, 2017 and 2018, aims to check whether this new regulatory system has improved transparency and objectivity in the criteria of distribution. The advertising insertions from the regional government (2016-2018) are examined and compared with the usual audience measurement metrics.
\end{abstract}

Key words: Institutional advertising, Legislation, Castilla-La Mancha, Media, Sectors.

Sumario. 1. Introducción. 2. Marco teórico y normativo. 2.1. Marco teórico. 2.2. Marco normativo. 3. Metodología. 4. El mapa del gasto en publicidad institucional. 5. La Publicidad institucional en Castilla-La Mancha. 5.1. El Acuerdo Marco como sistema regulador. 5.2. Evolución del Acuerdo Marco 2016-2018. 5.3. Distribución de importes publicitarios por soporte. 6. Discusión y conclusiones. 7. Referencias bibliográficas

\footnotetext{
Universidad de Castilla-La Mancha (España).

Universidad de Castilla-La Mancha (España).

Universidad de Castilla-La Mancha (España).
} 
Cómo citar: De la Cruz de Julián, J.; López-Cepeda, A.M.; Galletero-Campos, B. (2019) Sistemas regulatorios de la publicidad institucional y su eficacia: la Junta de Comunidades de Castilla-La Mancha como anunciante, en CIC. Cuadernos de Información y Comunicación, 135-153.

\section{Introducción}

La publicidad institucional es una modalidad de comunicación pública que tiene entre sus objetivos ofrecer información a los ciudadanos sobre cuestiones gubernamentales y construir la imagen de marca del Gobierno (Martínez Pastor, 2016). Su importancia como fuente de ingresos se justifica si tenemos en cuenta que algunos estudios (Cortés González, 2011; Martínez Pastor, 2012) han colocado al Estado como el primer anunciante del país en volumen presupuestario durante el periodo 2007-2009.

Las críticas a la publicidad institucional por la supuesta arbitrariedad no es una cuestión reciente. Ya se planteaba en los años 80, cuando los directivos de El Alcázar denunciaron la marginación a la que eran sometidos por parte del Gobierno de Felipe González (Edo, 1994:28); también en el 91 los diarios de Madrid reflejaron la controversia sobre el trato favorable hacia los medios de PRISA en las campañas gubernamentales (Edo, 1994:150). Más reciente es otro caso difundido en diversos medios, el de la campaña "No hay salida" del año 2012 realizada por el Ministerio de Sanidad dirigido por Ana Mato, cuyos datos, cruzados con las audiencias, mostraron algunas "desproporciones" favorables a medios afines al Partido Popular (Escolar, 2014).

A pesar de estas alusiones en la prensa, jurídicamente no es fácil demostrar a través de métricas que la distribución se hace por afinidades políticas o ideológicas. Sobre esta cuestión no existía doctrina jurídica del Tribunal Constitucional hasta el año 2014. La Sentencia 104/2014 constituyó así un punto de inflexión, ya que se trata de la primera de cuatro denuncias de Ser Castellón a varios ayuntamientos de la provincia (Castellón de la Plana, Villareal, Almazora y Burriana). La emisora provincial, líder de audiencia según las mediciones del Estudio General de Medios, fue excluida de la planificación publicitaria sin que hubiera ninguna justificación objetiva, tal y como reflejó la sentencia del Tribunal Constitucional. En ella no se negó que existiera una discriminación por razones ideológicas, pero sí se concluía que este hecho no se pudo acreditar, estimando que la consecución de dicha prueba era "altamente dificultosa". Ser Castellón no pudo demostrar que una de las causas de su exclusión en la contratación fuera la línea editorial, aunque ésta estuviera en clara discordancia con las administraciones contratantes 5 .

En Castilla-La Mancha no hay investigaciones previas centradas en el objeto de estudio, pero en 2014 varios medios de comunicación -incluido el diario público Lanza- se hicieron eco de un reportaje de ElPlural.com ${ }^{6}$ que detallaba cómo la de-

4 STC 104/2014 de 23 de junio, Ayuntamiento de Castellón de la Plana; STC 130/2014 de 21 de julio, Ayuntamiento de Villarreal; STC 147/2014 de 22 de septiembre, Ayuntamiento de Almazora; STC 160/2014 de 6 de octubre, Ayuntamiento de Burriana.

5 Contenido de la Sentencia 104/2014 (BOC NÚM 199/2014: 38).

6 "Destapamos los dineros opacos del 'Cospeleaks': 22 millones para los medios de comunicación amigos". ElPlural.com. 15/12/2014. Recuperado de: https://www.elplural.com/politica/destapamos-los-dineros-opacosdel-cospeleaks-22-millones-para-los-medios-de-comunicacion-amigos_34705102; 
nominada "Fundación para la promoción de Castilla-La Mancha", dependiente del ejecutivo regional presidido por María Dolores de Cospedal, habría invertido 22 millones de euros en publicidad institucional entre los años 2012 y 2014, montante que, según el diario digital, se habría adjudicado de forma discrecional entre los medios de comunicación, beneficiando explícitamente a aquellos más próximos ideológicamente al gobierno del PP. Entre los editores es frecuente reconocer la dependencia de este tipo de ingresos en un mercado caracterizado por los bajos índices de lectura de prensa y escaso tejido industrial (Galletero Campos, 2018). Sin embargo, no hay hasta el momento ningún trabajo científico en el que se analicen los importes invertidos por la Administración regional, la única que hace públicas estas asignaciones.

El objetivo general de este trabajo exploratorio es analizar la distribución de contratos publicitarios de la JCCM durante tres anualidades: 2016, 2017 y 2018 . Es una realidad que otras instituciones -ayuntamientos y diputaciones- realizan aportaciones económicas a los medios a través de campañas publicitarias, pero no publican las asignaciones, ni las proporcionan, aunque sean solicitadas, lo que obliga a circunscribir el estudio de caso al Gobierno autonómico. El propósito general se desglosa, a su vez, en tres objetivos específicos.

Objetivo 1. Presentar una comparativa en la inversión publicitaria de las administraciones regionales.

Objetivo 2. Examinar las inserciones publicitarias del Gobierno de Castilla-La Mancha en el periodo 2016-2018, de acuerdo a las siguientes variables: número de campañas, temática y soportes que se utilizan.

Objetivo 3. Comparar las métricas habituales de medición de audiencia (difusión, número de oyentes, visitas en OJD...) con los importes obtenidos por cada medio en los citados años.

\section{Marco teórico y normativo}

\subsection{Marco teórico}

La publicidad institucional, como publicidad del Estado, se puede identificar con el interés general (Martínez Pastor y Vizcaíno-Laorga, 2008), pero también como un instrumento persuasivo (Curto, 2013:111). De hecho, las posiciones críticas con este tipo de publicidad sostienen que, mediante los mensajes difundidos, puede encauzar la forma de afrontar las cuestiones de nuestra sociedad, de tal manera que se acabe produciendo una espiral del silencio (Noelle-Neuman, 1995) que afecta a los pensamientos discordantes (García López, 2001). En todo caso, coincidimos con Cortés González (2011) en afirmar que es pertinente analizar la inversión pública de acuerdo a la utilidad de las campañas y reflexionar sobre nuevos modelos comunicativos en un contexto en el que se cuestiona la unidireccionalidad de los mensajes en una concepción paternalista del Estado.

\footnotetext{
"El "Cospeleaks": "22 millones para los medios de comunicación amigos"”, Dclm.es, 15/12/2014. Recuperado de: https://www.dclm.es/noticias/29157/el-cospeleaks-22-millones-para-los-medios-de-comunicacion-amigos; "Elplural.com desvela que Cospedal ha inyectado más de 5 millones de euros al grupo de La Tribuna", Lanza Digital, 16/12/2014. Recuperado de: https:/www.lanzadigital.com/provincia/elplural-com-desvela-que-cospedal-ha-inyectado-mas-de-5-millones-de-euros-al-grupo-de-la-tribuna/
} 
La comunidad científica ha prestado cierta atención a este objeto de estudio, clasificándose los estudios localizados en cuatro áreas epistemológicas. 1) Desde una perspectiva teórica, varios autores se han interesado en el paso del concepto institucional desde el ámbito de la empresa privada al terreno público, directamente vinculado con la administración y la comunicación informativa (Alvarado y De Andrés, 2005; Pineda y Rey, 2009; Vilches, 2010). Otros se han ocupado de mostrar los límites y la relación entre publicidad institucional y la publicidad comercial o la propaganda (Ortega Martínez, 1999; García Uceda, 2001; Caro, 2008; Benavides 2010; Curto, 2013); 2) Son recurrentes los trabajos que abordan las campañas desde un punto de vista social, valorando las representaciones simbólicas (Gaona Martínez y Martínez Pastor, 2009; Martínez Pastor y Vizcaíno-Laorga, 2008) y el tratamiento y eficacia de los anuncios acerca de cuestiones como la seguridad vial (Segura García, 2015) o la violencia de género (Rodríguez López y Robles Álvarez, 2016). 3) Debido a la variedad de legislación, algunos análisis estudian el fenómeno desde un punto de vista normativo (Feliu Albaladejo, 2009) y judicial (Guichot, 2014); 4). Finalmente, se localizan investigaciones sobre distintas administraciones en calidad de anunciantes. Destacan los trabajos realizados por el Grupo de estudios sobre Publicidad Institucional y Social de la Universidad de Alicante, que ha llevado a cabo investigaciones sobre diversas comunidades autónomas: Valencia (Feliu García y Feliu Albaladejo, 2012), Galicia (Feliu García y Quintas, 2010), Cataluña (Feliu García y Feliu Albaladejo, 2011) y Andalucía (Fernández Poyatos y Feliu García, 2012). Más escasos son los que se refieren a administraciones de ámbito menor como los ayuntamientos (Ríos Martín, 2016). Este trabajo toma como base metodológica los estudios autonómicos, analizando la publicidad de la Junta de Comunidades de Castilla-La Mancha. Estos estudios de caso suponen un avance en la fiscalización de los diferentes sistemas de reparto y son posibles gracias a un contexto cada vez más exigente en la transparencia y rendición de cuentas.

Además de los cuatro grupos mencionados, se han revisado otros estudios que, no relacionados directamente con este concepto, sí abordan el sostenimiento económico por parte de las administraciones a los medios de comunicación. En este sentido, destacamos los trabajos sobre las subvenciones o ayudas directas que perciben algunos de ellos (Fernández Alonso y Blasco Gil, 2005; Blasco Gil, 2008; Galletero Campos y López Cepeda, 2018). Aunque la publicidad institucional es una acción comercial, regida por criterios de eficiencia económica, a menudo se convierte en un sistema de apoyo a los medios. De hecho, en el contexto europeo existen algunas políticas que así lo interpretan. En Italia, según el artículo 13 de la Ley del 5 de agosto de 1981, las administraciones estatales estaban obligadas a invertir en diarios y revistas una cuota superior al $70 \%$ de todo el gasto previsto para publicidad (Sanmartí, Aguado y Magallón, 2010). Otros autores sostienen que en Grecia también los anuncios gubernamentales suponen una fuente importante de ingresos (Psychogiopoulou, 2012; Van Besien, 2013). En Portugal, se ha considerado a los medios de comunicación sociales locales y regionales un vehículo idóneo para la difusión de publicidad institucional (Aguado, Sanmartí y Magallón, 2009).

Sin embargo, si no se realiza de acuerdo a criterios objetivos, este apoyo puede constituir una vía para favorecer a medios ideológicamente afines, interfiriendo en el equilibrio del libre mercado (Álvarez-Peralta y Franco, 2018). 


\subsection{Marco normativo}

Las campañas institucionales de publicidad y comunicación de la Administración General del Estado (AGE) se encuentran reguladas por la Ley 29/2005, de 29 de diciembre, de Publicidad y Comunicación Institucional, que se aprueba con el objetivo de garantizar la transparencia y la utilidad pública de las mismas. De acuerdo con esta norma, una campaña institucional es "toda actividad orientada y ordenada a la difusión de un mensaje u objetivo común, dirigida a una pluralidad de destinatarios, que utilice un soporte publicitario pagado o cedido y sea promovida o contratada por la Administración General del Estado y por las demás entidades integrantes del sector público estatal" (Ley 28/2005, art.2). Además, por su naturaleza, las licitaciones de estas acciones están reguladas por la Ley 9/2017, de 8 de noviembre, de Contratos del Sector Público que introduce los requisitos para la formalización de contratos en los que una de las partes sea la Administración Pública. Estas transacciones están sometidas a principios de transparencia, establecidos por la Ley 19/2013, de 9 de diciembre, de transparencia, acceso a la información pública y buen gobierno, que ha situado a nuestro país a la altura de la mayoría de los países europeos que ya tenían en vigor una legislación similar.

Además de la legislación nacional que afecta a las actuaciones de la AGE, los planes institucionales de publicidad de ámbito regional son asumidos desde el año 2000 por los Ejecutivos autonómicos (Colino, 2013). Doce comunidades autónomas han desarrollado legislación autonómica sobre la materia, siendo la de Cataluña, promulgada en diciembre de 2000, la más antigua entre las vigentes. A lo largo de más de una década varios parlamentos regionales han aprobado normativas, en orden cronológico: Comunidad Valenciana (2003), Aragón (2003), Navarra (2003), Andalucía (2005), Comunidad de Madrid (2005), Asturias (2006), Canarias (2007), Castilla y León (2009), Baleares (2010) y País Vasco (2010). Las últimas que han desarrollado una norma específica han sido Extremadura (2013) y La Rioja (2017) (Galletero Campos y López-Cepeda, 2018).

En Castilla-La Mancha aún no se ha aprobado una legislación sobre la materia, pero en 2016 el Ejecutivo autonómico, a través de la Dirección General de Promoción Institucional, promovió un Acuerdo Marco con el objetivo de "racionalizar, ordenar y adoptar los tipos contratables relativos a la compra de espacios publicitarios con empresas titulares de medios de comunicación”. En la justificación del documento, la JCCM enuncia la necesidad de dar cobertura informativa y publicitaria a la actividad de la Administración regional. Este proceso de contratación ha supuesto una novedad en el mapa de la publicidad institucional a nivel estatal, pues se produce dentro de una comunidad autónoma que no tiene una propia ley de publicidad institucional y se trata de un modelo nuevo para la adjudicación de los contratos.

Aunque la fórmula del Acuerdo Marco es utilizada por la Administración General del Estado desde 2014, la propuesta castellanomanchega sí supone una innovación en cuanto a quién va dirigido: mientras que la administración estatal adjudica las campañas a agencias de medios, que a su vez son las encargadas de negociar las inserciones publicitarias con las empresas de comunicación, en el caso de la JCCM los acuerdos se conciertan directamente con los medios. Por lo específico del modelo y por la ausencia de estudios en este ámbito territorial, consideramos de relevancia el análisis una Comunidad Autónoma en donde el gasto en publicidad institucional ha 
pasado de cuatro millones de euros $(4.082 .326,48)$ en el año 2016 hasta más de seis millones en el año $2018(6.269 .174,26)$.

\section{Metodología}

Los criterios de adjudicación de publicidad institucional establecidos en el Acuerdo Marco de Castilla-La Mancha se basan en dos variables, con un valor de un $50 \%$ cada una, para ponderar la puntuación de las ofertas: una de ellas, de tipo cuantitativo, es el coste del anuncio según el formato establecido (lugar de exposición y duración de la publicidad); y la otra, de tipo cualitativo, se basa en las propuestas de mejora que realicen los medios y que permitan una mayor eficacia para las campañas.

A través de la Plataforma de Contratación del Sector Público se han obtenido los importes correspondientes a cada campaña y a cada medio de comunicación para las tres anualidades analizadas. Una de las dificultades es que la relación de nombres que figura en este portal es la de la empresa editora (a veces incluso el nombre de un trabajador por cuenta propia), por lo que hay que acudir al Registro Mercantil o al medio de comunicación para indagar en la propiedad de cada medio. Una vez desglosados y asignados los importes, se han revisado distintos indicadores de audiencias de los medios castellano-manchegos, tales como los reflejados en la Oficina de Justificación de la Difusión, el EGM (Estudio General de Medios) y en OJD interactiva, con el fin de detectar si se producen algunas desviaciones o anomalías sobre los importes concedidos a tenor de la posición competitiva del medio. Aunque la audiencia no es un indicador que se mencione de manera específica en el Acuerdo Marco, es la base sobre la que los medios establecen sus tarifas publicitarias (uno de los dos criterios contemplados). Además, la Junta de Comunidades indica en el Acuerdo que "se diseña el plan de medios para optimizar la inversión, mediante la maximización de la presión en el público objetivo, de tal manera que los anuncios impacten a todos los ciudadanos de Castilla-La Mancha posibles" (JCCM, 2016: 5).

\section{El mapa del gasto en publicidad institucional}

Cuatro son las comunidades autónomas -Castilla-La Mancha, Murcia, Navarra y Cantabria- que, aun no disponiendo de una ley específica sobre Publicidad y Comunicación Institucional, sí han desarrollado dentro de las respectivas leyes de Transparencia artículos que regulan la materia de forma concreta. Se produce la paradoja de que en estas cuatro regiones los datos referentes a la publicidad institucional son accesibles; en cambio, en otras como Canarias, Baleares o País Vasco, con leyes específicas de publicidad institucional, los mismos datos no se publican de una manera que permita analizarlos. Cotejando la información de los portales autonómicos con los presupuestos generales aprobados para 2017 para las diecisiete comunidades, se ha realizado una comparativa que recoge la inversión en este tipo de campañas por cada 10.000 euros de gasto público. Como se puede ver en la tabla 1, son Cataluña y Andalucía las comunidades con mayor inversión publicitaria, llegando la primera de ellas - con 30 millones de euros a superar la inversión de la Administración General 
del Estado, 27 millones de euros en 2017 (Ministerio de la Presidencia, 2017). Por otro lado, en relación a su presupuesto global, destaca la ratio de inversión de $\mathrm{La}$ Rioja, a la cabeza, a pesar de que es la que tiene menor número de habitantes del conjunto de comunidades con datos accesibles. Por otro lado, Castilla y León, con una población similar a la de Castilla-La Mancha, dobla la inversión en publicidad institucional. La autonomía castellanomanchega se sitúa así en la mitad inferior de la tabla, con un volumen per cápita similar al de Extremadura.

Tabla 1 y gráfico 1. Gasto de las Comunidades Autónomas en Publicidad Institucional sobre presupuesto.

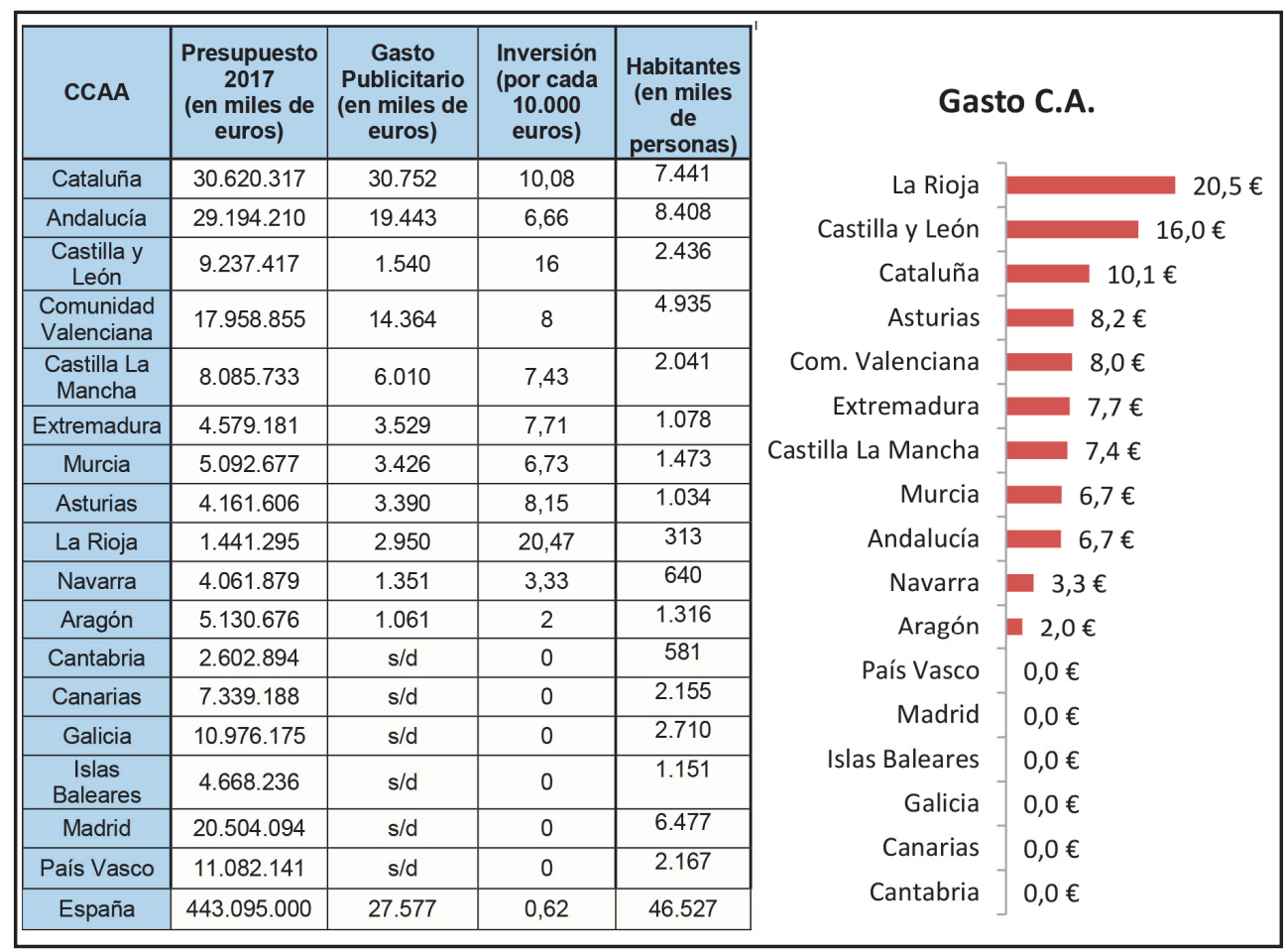

Fuente: Elaboración propia a partir de los portales de transparencia autonómicos, presupuestos autonómicos recogidos en la en la página web del Ministerio de Hacienda e INE. El gasto C. A. se refiere al gasto per cápita.

\section{La Publicidad institucional en Castilla-La Mancha}

\subsection{El Acuerdo Marco como sistema regulador}

El Acuerdo Marco se adoptó en el año 2016 para "homogeneizar y simplificar los procedimientos de contratación y lograr mayores niveles de seguridad, publicidad y transparencia mediante la celebración de un único procedimiento de homologación abierto a la concurrencia y a la competencia" (JCCM, 2016: 11). Su finalidad es simplificar y reducir costes, al constar de un único episodio de concurrencia por parte de los posibles contratistas. Por su parte, los medios tienen que reunir varios 
requisitos para formar parte del precontrato que supone el AM, como tener solvencia económica o capacidad de contratar con la administración según la Ley de Contratos del Sector Público. Además, tienen la obligación de poner a disposición de la JCCM datos sobre la monitorización de las audiencias a través de sistemas como el Estudio General de Medios para televisiones y radios, la Oficina de Justificación de Difusión y OJD Interactiva o ComScore para las mediciones de los digitales. Para preservar la profesionalidad del medio también es obligatorio que dentro de la empresa adjudicataria figure una persona, sea como propietario o empleado, graduada o licenciada en Periodismo, cuya titulación se debe acreditar en la solicitud.

\subsection{Evolución del Acuerdo Marco 2016-2018}

Los resultados del análisis de los datos del Portal de Transparencia revelan que durante los últimos tres años la JCCM ha realizado un total de 81 campañas: 39 en 2016, 42 en 2017 y 47 en 2018. A lo largo de este tiempo, el gasto en publicidad institucional ha pasado de 4.082.326,48 euros a 6.269.174,26 euros, lo que supone un incremento del 53,57\%. Para clasificar las campañas, se han considerado cuatro categorías, en línea con la tipificación propuesta por Feliu Albaladejo y Feliu García (2012):

- Social: Campañas encaminadas a promover valores y visualizar temas relevantes como la discriminación y violencia de género, la prevención de incendios o asuntos de interés sanitario.

- Económico: Propuestas de índole comercial como la promoción del tejido empresarial, incidiendo en sectores capitales para Castilla-La Mancha como la alimentación y la artesanía. En este apartado se incluyen aquellas que buscan el fomento de la oferta turística.

- Cultural: Campañas que tienen como objetivo ofrecer y poner en valor la actividad cultural patrocinada por la JCCM, además de visibilizar las costumbres propias.

- Corporativo: Dirigidas a difundir los servicios públicos de la administración autonómica, dar notoriedad y mejorar la propia imagen de los órganos dependientes de la Junta, crear una imagen de marca y reforzar la propia identidad castellano manchega entre sus habitantes.

Como se puede apreciar, la mayoría de las campañas se refieren a aspectos económicos, lo que evidencia la importancia que supone el apoyo de la Administración en una región donde el sector servicios y el turismo son los principales pilares del PIB. Es relevante el incremento producido en las campañas de tipo social, ya que dentro del periodo de estudio se ha duplicado el número de campañas (de 7 a 14), así como el dinero invertido, que ha pasado de 987.112,5 euros en 2016 a 2.184.738 en 2018. Entre estas, se encuentran, por ejemplo, la del Día Mundial del Agua, el Día Internacional de las Mujeres, el uso racional de los medicamentos o la concienciación sobre el riesgo de incendios. 
Gráfico 2. Número de campañas contratadas por la JCCM.

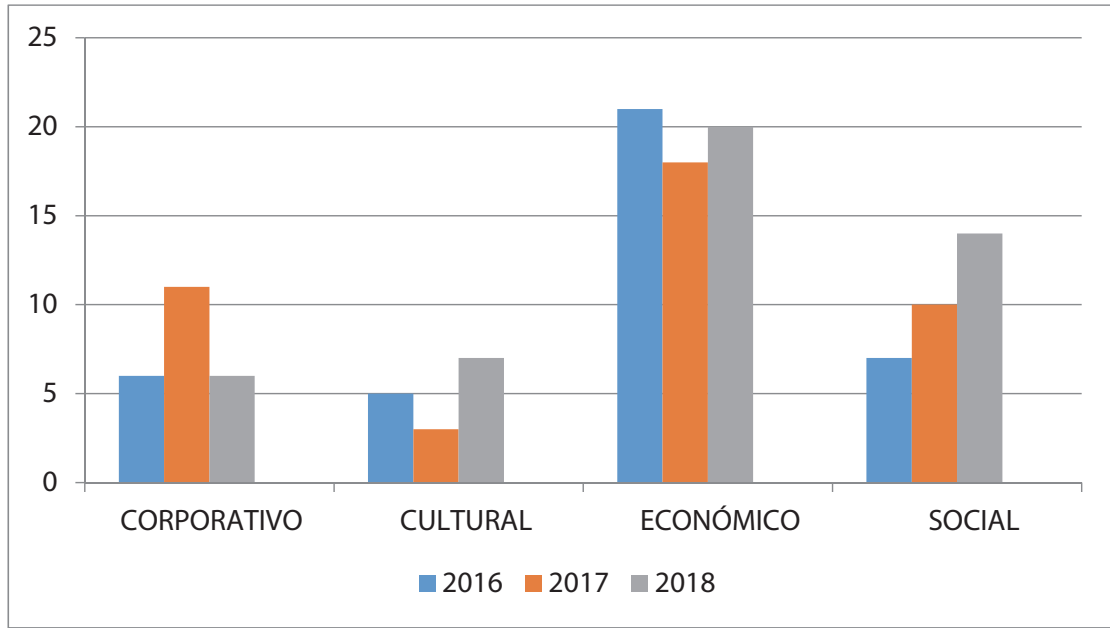

Fuente: Elaboración propia a partir de datos del Portal de Transparencia de Castilla-La Mancha.

\subsection{Distribución de importes publicitarios por soporte}

Las empresas de comunicación interesadas en participar en este Acuerdo Marco en el momento de su lanzamiento fueron 109 , siendo dos de ellas rechazadas por no reunir las condiciones. Si nos fijamos en el número de medios, durante las tres anualidades se han formalizado contratos con un total de 239 medios, la mayor parte de ellos de cobertura regional, aunque en diversas campañas se insertó publicidad en webs y prensa escrita de difusión nacional. Según el Informe sobre los medios de Comunicación en Castilla-La Mancha 2015 (Laguna et al., 2016), el número de medios generalistas en Castilla-La Mancha el año de su puesta en marcha era de 144, lo que refuerza la tesis de la importancia de la publicidad institucional en el mercado castellanomanchego de la comunicación. Los medios se han clasificado en agencias de noticias, medios digitales, publicaciones impresas, emisoras de radio y televisiones.

Tabla 2. Volumen de inversión en número de campañas e importes (2016-2018).

\begin{tabular}{|l|l|l|l|l|l|l|}
\hline \multirow{2}{*}{\begin{tabular}{c}
\multirow{2}{*}{$\begin{array}{c}\text { TIPO DE } \\
\text { MEDIOS }\end{array}$} \\
\multirow{2}{*}{ NÚM }
\end{tabular}} & \multicolumn{4}{|c|}{ IMPORTE } & \multirow{2}{*}{ PORCENTAJE } \\
\cline { 3 - 6 } & & $\mathbf{2 0 1 6}$ & $\mathbf{2 0 1 7}$ & $\mathbf{2 0 1 8}$ & \multicolumn{1}{c|}{ Total } & \\
\hline AGENCIAS & 1 & 363 & 19.501 & 15.237 & 35.101 & $0,21 \%$ \\
\hline $\begin{array}{l}\text { MEDIOS } \\
\text { DIGITALES }\end{array}$ & 85 & 892.548 & 956.803 & 1.239 .035 & 3.088 .386 & $18,88 \%$ \\
\hline PUB. IMPRESAS & 84 & 1.224 .480 & 2.482 .002 & 2.285 .598 & 5.992 .080 & $36,62 \%$ \\
\hline TELEVISIÓN & 16 & 859.768 & 1.009 .912 & 1.118 .512 & 2.988 .192 & $18,26 \%$ \\
\hline TOTAL & 239 & 4.082 .326 & 6.009 .632 & 6.269 .175 & 16.361 .133 & \\
\hline
\end{tabular}

Fuente: Elaboración propia a partir de los datos del Acuerdo Marco. 
La distribución de la publicidad en función del soporte revela que, en número de medios, el primer lugar lo ocupan los medios digitales. Sin embargo, en cuanto a gasto de contratación, el número más elevado es el de las publicaciones impresas (periódicos diarios, semanarios y revistas), lo que guarda relación con unos precios por inserción publicitaria más elevados. Algo similar ocurre en el caso de las televisiones; un número reducido de ellas han alcanzado el 18,26\% del importe global. Esta tendencia contrasta con la reciente "digitalización de la inversión" (ÁlvarezPeralta y Franco, 2018) que se viene produciendo en la publicidad del Estado. En este caso, el apoyo hacia los medios más tradicionales se hace evidente en la distribución porcentual de las cuantías.

\subsubsection{Medios digitales}

En la adjudicación de la publicidad institucional a los medios digitales se observa que no solo se aplica el criterio de mayor audiencia, tal y como se preveía tras la lectura del Acuerdo Marco y el Pliego de Condiciones. Los medios que tienen más visitas no son los que reciben los contratos más cuantiosos: La Cerca.com, Eldigitalcastillalamancha.es, clm24.es o eldigitaldealbacete.com son los medios que con diferencia reciben más visitas en sus webs; no obstante, no encabezan el ranking de aquellos que más dinero han percibido. En el otro extremo, webs como encastillalamancha.es o dclm.es han ingresado importes de una cuantía superior a la que les debería corresponder teniendo en cuenta solamente los datos de audiencia monitorizados. La edición de eldiario.es para Castilla-La Mancha es el segundo medio en volumen de inversión; sin embargo, ocupa la décima posición en número de visitas en diciembre de 2018. Estos datos confirman que no siempre existe una relación directa entre el número de lectores y las cuantías percibidas, sino que intervienen también otros factores como la visibilidad del espacio ofertado (15 puntos), la ubicación del anuncio respecto al resto de anunciantes (10 puntos), la calidad del contenido junto al que se publicita (5 puntos) y formatos especiales como reportajes publicitarios, branded content o menciones en redes sociales, entre otras (20 puntos)" (JCCM, 2016: 29). En este caso, las puntuaciones en cada uno de estos apartados no son accesibles.

Aunque son 85 los medios digitales que han suscrito contratos con la JCCM, sólo los ocho primeros suman más del $70 \%$ del volumen total de inversión, siendo todos ellos de ámbito autonómico excepto El Digital de Albacete. En el desglose de los importes, se advierte que publicaciones impresas perciben cuantías diferentes por sus anuncios en la versión impresa y en la digital, lo que favorece que una misma empresa acumule importes muy elevados, tal y como sucede con el grupo Promecal, propietario de las Tribunas provinciales. Esta situación, en cambio, no se produce con las webs de las emisoras de radio o de las televisiones.

Sobre la eficacia del sistema para fomentar la transparencia, se puede señalar que el número de medios digitales que se han incorporado a las mediciones OJD Interactiva ha crecido de manera exponencial a partir de la publicación del Acuerdo Marco (Gráfico 4), al constituir estos datos un requisito obligatorio para suscribir un contrato. El mayor incremento se produce en el último trimestre de 2016, alcanzándose cierta estabilidad a lo largo de 2018. 
Tabla 3. Ranking de medios digitales por importe (importes superiores a 10.000 euros).

\begin{tabular}{|c|c|c|c|c|c|c|c|c|c|c|c|c|c|c|c|c|c|c|c|c|}
\hline & 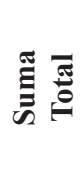 & 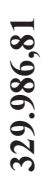 & $\begin{array}{l}\hat{b} \\
\hat{a} \\
\dot{J} \\
\stackrel{0}{0} \\
\stackrel{N}{N}\end{array}$ & 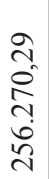 & $\begin{array}{c}2 \\
2 \\
n \\
0 \\
n \\
\infty \\
n \\
n\end{array}$ & $\begin{array}{l}\stackrel{\sim}{n} \\
\infty \\
\stackrel{n}{n} \\
\stackrel{\sim}{N}\end{array}$ & 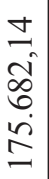 & $\begin{array}{l}\vec{b} \\
\Omega \\
\hat{\gamma} \\
\hat{\sigma} \\
\end{array}$ & 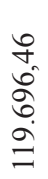 & $\begin{array}{l}\hat{o} \\
\hat{R} \\
\hat{2} \\
\dot{a}\end{array}$ & 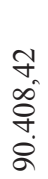 & 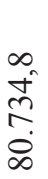 & 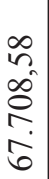 & $\mid \begin{array}{c}0 \\
\tilde{0} \\
2 \\
0 \\
\dot{0} \\
0\end{array}$ & $\frac{\mathfrak{N}}{\stackrel{a}{\sigma}}$ & $\begin{array}{l}\infty \\
0 \\
\hat{\infty} \\
\tilde{n} \\
i \\
i\end{array}$ & $\begin{array}{c}0 \\
\bar{b} \\
\dot{0} \\
\infty \\
\dot{\vec{n}} \\
n\end{array}$ & $\begin{array}{l}\frac{\infty}{2} \\
\stackrel{-}{\infty} \\
\infty \\
\dot{\sigma}\end{array}$ & 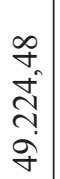 & 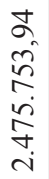 \\
\hline \multirow{2}{*}{$\stackrel{\infty}{\bar{N}}$} & $\overbrace{\overline{0}}^{\infty}$ & 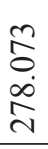 & 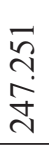 & 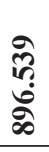 & 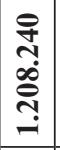 & $\begin{array}{l}\circ \\
80 \\
\dot{0} \\
\infty \\
\infty \\
-\end{array}$ & $\begin{array}{l}\stackrel{0}{\sigma} \\
\infty \\
\dot{0} \\
\stackrel{\sim}{N}\end{array}$ & $\begin{array}{l}0 \\
+ \\
\infty \\
0 \\
0 \\
0\end{array}$ & $\begin{array}{l}\stackrel{8}{0} \\
\infty \\
\dot{H}\end{array}$ & $\begin{array}{l}\tilde{n} \\
\tilde{n} \\
\tilde{N}\end{array}$ & 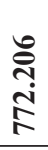 & \begin{tabular}{l}
$\infty$ \\
$\stackrel{2}{0}$ \\
ஸे \\
$\infty$ \\
\multirow{+}{+}{}
\end{tabular} & \begin{tabular}{l}
\multirow{2}{*}{} \\
6 \\
$\infty$ \\
$\infty$ \\
$n$
\end{tabular} & $\begin{array}{l}0 \\
+ \\
\infty \\
\infty \\
0 \\
-\end{array}$ & 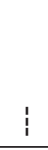 & 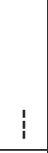 & $\frac{a}{\sim}$ & $\begin{array}{l}\stackrel{n}{N} \\
\stackrel{N}{N}\end{array}$ & 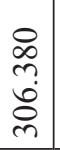 & \\
\hline & 泀 & 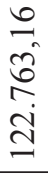 & $\begin{array}{l}\hat{ర} \\
\text { ปิ } \\
\delta \\
\infty \\
i \\
=\end{array}$ & 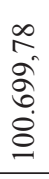 & $\begin{array}{c}\hat{\infty} \\
0 \\
\tilde{\sigma} \\
\mathfrak{n} \\
ٍ \\
=\end{array}$ & $\begin{array}{l}\bar{a} \\
\text { aे } \\
\dot{\sigma} \\
\dot{\sigma}\end{array}$ & $\begin{array}{l}\hat{N} \\
\hat{s} \\
\dot{+} \\
\infty \\
\infty\end{array}$ & 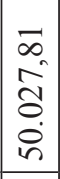 & 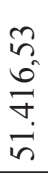 & $\begin{array}{l}\hat{\sigma} \\
\infty \\
\hat{\sigma} \\
\infty \\
\infty \\
+\end{array}$ & 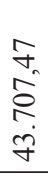 & 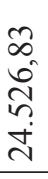 & $\begin{array}{l}\infty \\
n \\
\Xi \\
\infty \\
\dot{\sim} \\
\sim\end{array}$ & 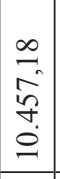 & $\begin{array}{l}a \\
\tilde{n} \\
\underline{n} \\
=\end{array}$ & $\begin{array}{l}n \\
0 \\
-1 \\
\dot{0} \\
\sim\end{array}$ & $\begin{array}{l}0 \\
0 \\
n \\
\ddots \\
0 \\
\ddots\end{array}$ & 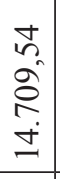 & $\begin{array}{l}\tilde{m} \\
m \\
m \\
m \\
m\end{array}$ & $\begin{array}{l}2 \\
\infty \\
0 \\
\infty \\
\infty \\
0 \\
\infty \\
\infty\end{array}$ \\
\hline & 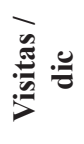 & $\begin{array}{l}\text { જ̆ } \\
\text { ஸ் }\end{array}$ & $\begin{array}{l}\exists \\
\mathfrak{n} \\
0\end{array}$ & 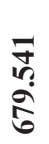 & $\begin{array}{l}\overline{0} \\
\dot{0} \\
\dot{0} \\
0 \\
0\end{array}$ & 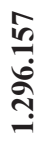 & $\begin{array}{l}\stackrel{a}{+} \\
\stackrel{\sim}{N} \\
\text { ते }\end{array}$ & 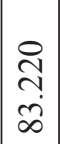 & $\begin{array}{l}\text { D } \\
\stackrel{\infty}{+} \\
\text { in }\end{array}$ & $\begin{array}{l}m \\
\infty \\
\cdots \\
\stackrel{N}{0}\end{array}$ & 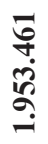 & $\frac{\stackrel{n}{c}}{\stackrel{\infty}{\infty}}$ & $\begin{array}{l}\stackrel{J}{J} \\
\stackrel{+}{ \pm} \\
\vec{J}\end{array}$ & 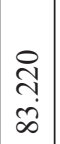 & 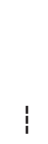 & $\mid$ & 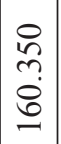 & $\overrightarrow{\hat{\sigma}}$ & $\vec{\sigma}$ & \\
\hline & & $\begin{array}{l}\vec{\infty} \\
\vec{i} \\
\tilde{n} \\
\text { di }\end{array}$ & 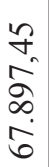 & 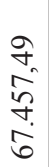 & $\begin{array}{l}n \\
\hat{\delta} \\
+ \\
\dot{0} \\
\infty\end{array}$ & $\begin{array}{c}\infty \\
\stackrel{0}{0} \\
0 \\
0 \\
\frac{1}{\infty}\end{array}$ & 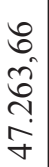 & 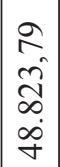 & $\frac{2}{2}$ & 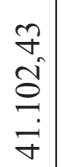 & 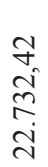 & 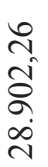 & 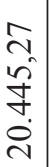 & 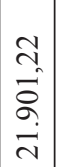 & 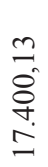 & 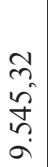 & $\begin{array}{c}\infty \\
0 \\
a \\
a \\
\tilde{a} \\
\tilde{v}\end{array}$ & $\begin{array}{l}n \\
\ddot{n} \\
\dot{\gamma} \\
\dot{v}\end{array}$ & $\begin{array}{l}\hat{\sigma} \\
\tilde{N} \\
\hat{o} \\
\tilde{a}\end{array}$ & $\begin{array}{l}n \\
n \\
0 \\
0 \\
0 \\
8 \\
\therefore\end{array}$ \\
\hline & 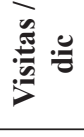 & $\begin{array}{l}\hat{\infty} \\
0 \\
0 \\
0\end{array}$ & $\begin{array}{l}\infty \\
\infty \\
\dot{\sigma} \\
=\end{array}$ & $\begin{array}{l}n \\
n \\
n \\
2 \\
2\end{array}$ & 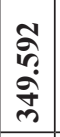 & 赵 & 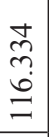 & 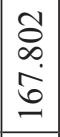 & $\begin{array}{l}\vec{\infty} \\
\stackrel{\infty}{\infty} \\
\stackrel{N}{ }\end{array}$ & 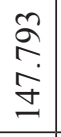 & $\begin{array}{l}\underset{\infty}{\infty} \\
\stackrel{+}{\infty} \\
\infty \\
\sigma\end{array}$ & \begin{tabular}{l}
$\infty$ \\
\multirow{N}{*}{} \\
$\substack{\infty \\
+}$
\end{tabular} & $\vdots$ & $\begin{array}{l}0 \\
\delta \\
\infty \\
\hat{0} \\
-1 \\
\end{array}$ & 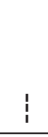 & $!$ & $\begin{array}{l}a \\
\dot{\sigma} \\
\stackrel{ }{=}\end{array}$ & $\begin{array}{l}8 \\
\infty \\
\infty \\
\infty \\
0 \\
0\end{array}$ & $\begin{array}{l}8 \\
0 \\
0 \\
0 \\
0 \\
-1\end{array}$ & \\
\hline & 䒾 & $\begin{array}{l}\stackrel{+}{\infty} \\
\stackrel{0}{\circ} \\
\dot{0}\end{array}$ & $\begin{array}{l}n \\
\frac{n}{2} \\
\frac{2}{0} \\
a\end{array}$ & $\begin{array}{l}\widetilde{J} \\
\stackrel{ }{0} \\
= \\
\infty \\
\infty\end{array}$ & 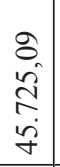 & 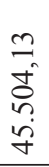 & $\begin{array}{c}\vec{\sim} \\
\Xi \\
\bar{\vdots} \\
i \\
n\end{array}$ & 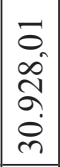 & 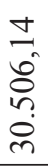 & $\begin{array}{l}n \\
n \\
\hat{\sigma} \\
a \\
a\end{array}$ & 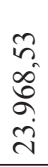 & 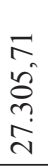 & 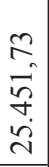 & $\begin{array}{l}m \\
\tilde{N} \\
m \\
\tilde{r} \\
m\end{array} \mid$ & 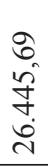 & 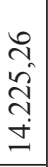 & $\begin{array}{l}\stackrel{\sim}{f}_{0} \\
\underset{\Xi}{\infty} \\
\Xi \\
=\end{array}$ & $\begin{array}{l}\exists \\
\tilde{n} \\
\sigma \\
= \\
=\end{array}$ & 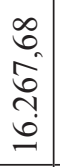 & 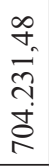 \\
\hline & 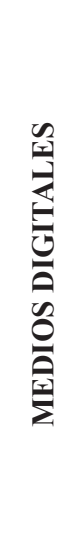 & 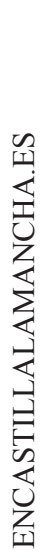 & 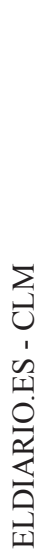 & 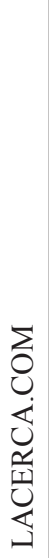 & 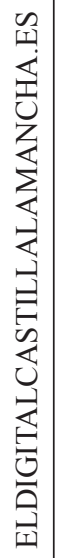 & 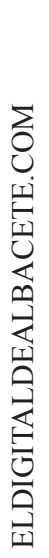 & 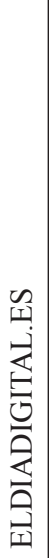 & 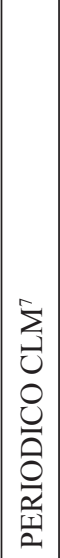 & 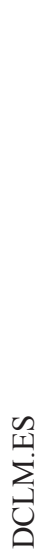 & 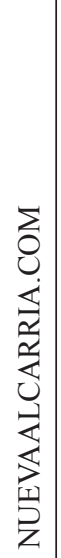 & 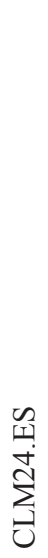 & 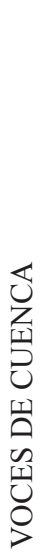 & 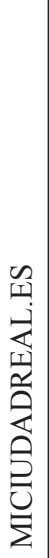 & $\begin{array}{c}n \\
1 \\
0 \\
0 \\
0 \\
0 \\
0 \\
0 \\
0 \\
0\end{array}$ & 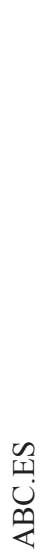 & 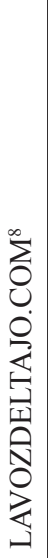 & 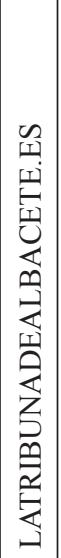 & 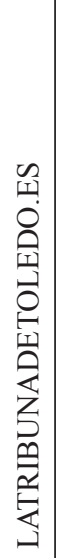 & 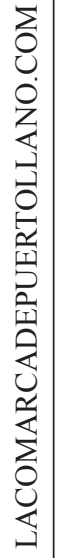 & 岕 \\
\hline
\end{tabular}

7 Periódico CLM y Público.es figuran como un mismo medio en OJD Interactiva, por eso las audiencias señaladas son iguales; sin embargo, a efectos de contratación de campañas, aparecen como empresas independientes. Digital no auditado por OJD Interactiva. 
Gráfico 3. Evolución de medios digitales castellanomanchegos auditados por OJD Interactiva.

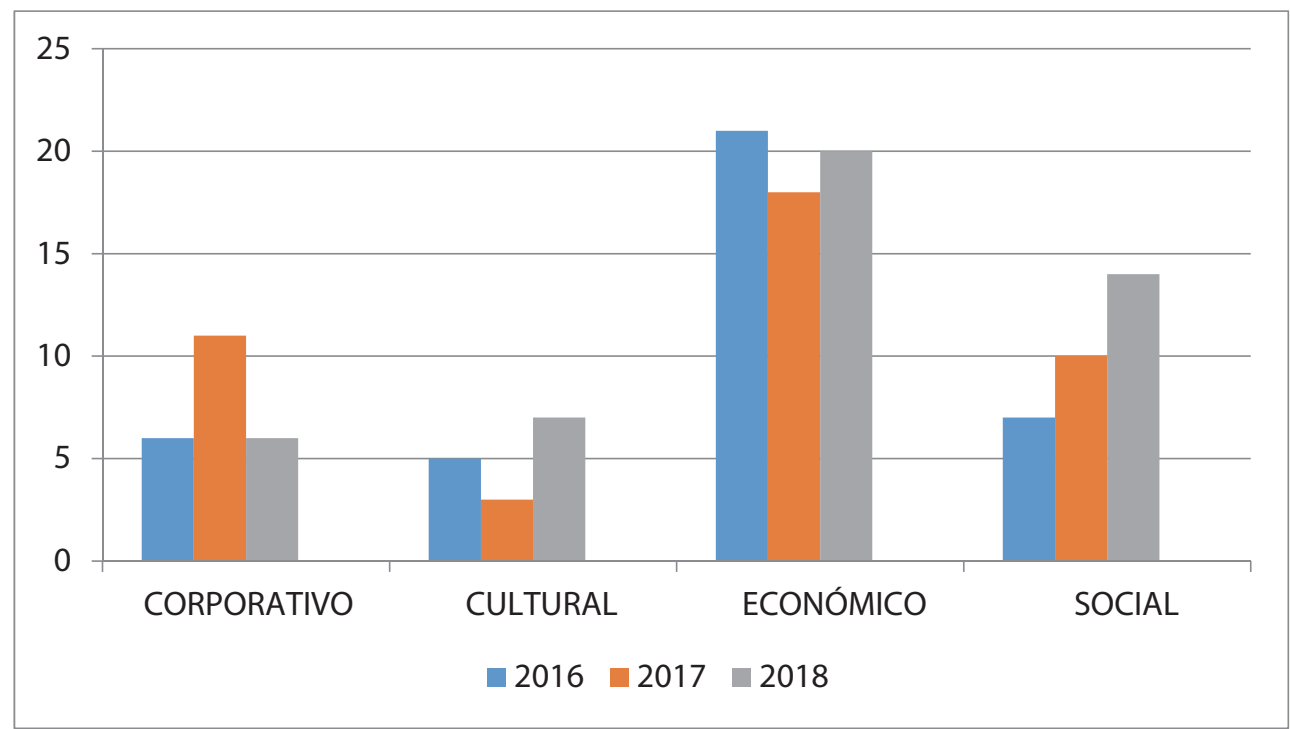

Fuente: Elaboración propia a partir de los datos publicados en OJD Interactiva.

Sin embargo, hay que notar que cabe mayor exigencia en lo que se refiere al cumplimiento de las condiciones exigidas en el artículo 71 de la Ley de Contratos del Sector Público, ya que una de las empresas concurrentes se encuentra desde abril de 2016 en situación de concurso voluntario de acreedores ${ }^{9}$. Esta circunstancia impediría, de acuerdo a la citada normativa, suscribir contratos con la administración.

\subsubsection{Publicaciones impresas}

En relación a la prensa impresa, el dato más llamativo se refiere al hecho de que a lo largo de los tres años se han realizado inserciones por más de 282.000 euros en ocho medios escritos cuyo ámbito de difusión se circunscribe a la comunidad de Castilla y León, un hecho que no parece justificarse con la vocación del plan de medios de alcanzar al mayor número de ciudadanos castellanomanchegos. Estos ocho medios (El Día de Segovia, El Día de Valladolid, El Día de Salamanca, El Día de León, El Día de Soria, Diario de Ávila, Diario de Burgos y Diario Palentino) pertenecen al grupo Promecal, propietario a su vez de las cabeceras impresas y digitales de las Tribunas de Albacete, Toledo, Ciudad Real (diarios), y Cuenca y Guadalajara (semanarios). Así, el grupo empresarial ha sido adjudicatario de contratos en trece medios impresos (la totalidad de sus medios de prensa escrita en 2017) ${ }^{10}$ y cuatro medios

\footnotetext{
9 Declaración recogida en el BOE con fecha de 19 de mayo de 2016. https://www.boe.es/boe/dias/2016/05/19/ pdfs/BOE-B-2016-21144.pdf

10 Actualmente el grupo de comunicación PROMECAL tiene sólo once cabeceras provinciales ya que El Día de León y El Día de Salamanca cerraron en abril de 2018.
} 
digitales, por un importe de más de dos millones y medio de euros, lo que supone la quinta parte de la inversión en publicidad institucional realizada por el gobierno castellano-manchego.

Por otro lado, se puede reseñar la desigualdad en las inversiones percibidas por algunos semanarios provinciales. Mientras que Las Noticias de Cuenca (171.754,77 euros), La Tribuna de Cuenca (211.994,37 euros) y La Voz del Tajo (227.420,28 euros) han percibido cuantías en torno a los 200.000 euros y Nueva Alcarria, que también tiene versión impresa, ha superado los 540.000, otros semanarios como El Semanal de la Mancha se queda en poco más de 45.000 euros, con una tirada similar a la de otras publicaciones citadas. La Tribuna de Cuenca pertenece, como se ha señalado, a Promecal; el semanario Nueva Alcarria está editado por Editorial Nueva Alcarria, S.A., sociedad dentro del grupo Quabit Inmobiliaria, S.A. En cambio, Las Noticias de Cuenca, La Voz del Tajo y El Semanal de la Mancha están en manos de pequeñas empresas editoras de ámbito local, Ediciones y Servicios Integrales 2020 S.L, Tajo Media S.L. y Ediciones Centro Mancha S. L., respectivamente.

Otro dato llamativo es el elevado importe percibido por el diario $A B C$, cuyas campañas se asignan por separado a la edición nacional y a la regional, a pesar de que esta última consiste en unas páginas diferenciadas insertas en el diario nacional. En términos generales, seis publicaciones (dos de ellas de ámbito nacional) concentran tres cuartas partes de la inversión.

Tabla 4. Ranking de medios impresos por importe recibido (importes superiores a 30.000 euros en total).

\begin{tabular}{|l|l|l|l|l|l|}
\hline Publicaciones impresas & \multicolumn{1}{|c|}{$\mathbf{2 0 1 6}$} & $\mathbf{2 0 1 7}$ & $\mathbf{2 0 1 8}$ & \multicolumn{1}{|c|}{$\begin{array}{c}\text { Suma } \\
\text { Total }\end{array}$} & $\begin{array}{c}\text { Difusión } \\
\text { en } \\
\text { C-LM } \\
\mathbf{( 2 0 1 7 )}\end{array}$ \\
\hline $\begin{array}{l}\text { LA TRIBUNA DE } \\
\text { TOLEDO }\end{array}$ & $131.122,08$ & $384.205,28$ & $297.133,41$ & $812.460,77$ & 2.006 \\
\hline $\begin{array}{l}\text { LA TRIBUNA DE } \\
\text { CIUDAD REAL }\end{array}$ & $123.538,55$ & $352.634,06$ & $294.192,14$ & $770.364,75$ & 2.065 \\
\hline $\begin{array}{l}\text { LA TRIBUNA DE } \\
\text { ALBACETE }\end{array}$ & $112.503,23$ & $305.030,59$ & $275.523,05$ & $693.056,87$ & 2.898 \\
\hline ABC CLM & $135.164,68$ & $324.576,02$ & $227.868,91$ & $687.609,61$ & $3.873 *$ \\
\hline NUEVA ALCARRIA & $111.240,71$ & $208.071,6$ & $221.628,44$ & $540.940,75$ & 2.989 \\
\hline EL PAIS & $71.670,21$ & $101.081,52$ & 62.315 & $235.066,73$ & $4.232 *$ \\
\hline LA VOZ DEL TAJO & $79.127,4$ & $62.864,34$ & $85.428,54$ & $227.420,28$ & 3.003 \\
\hline $\begin{array}{l}\text { LA TRIBUNA DE } \\
\text { CUENCA }\end{array}$ & $40.551,89$ & $68.205,28$ & $10.3237,2$ & $211.994,37$ & s.d. \\
\hline $\begin{array}{l}\text { LAS NOTICIAS DE } \\
\text { CUENCA }\end{array}$ & $72.133,05$ & 39.809 & $59.812,72$ & $171.754,77$ & 992 \\
\hline
\end{tabular}




\begin{tabular}{|l|l|l|l|l|l|}
\hline Publicaciones impresas & \multicolumn{1}{|c|}{$\mathbf{2 0 1 6}$} & \multicolumn{1}{|c|}{$\mathbf{2 0 1 7}$} & $\mathbf{2 0 1 8}$ & \multicolumn{1}{|c|}{$\begin{array}{c}\text { Suma } \\
\text { Total }\end{array}$} & $\begin{array}{l}\text { Difusión } \\
\text { en } \\
\text { C-LM } \\
\text { (2017) }\end{array}$ \\
\hline $\begin{array}{l}\text { LA TRIBUNA DE } \\
\text { GUADALAJARA }\end{array}$ & $20.473,2$ & $65.882,08$ & $80.331,9$ & $166.687,18$ & s.d. \\
\hline DIARIO DE AVILA & - & $77.751,7$ & $31.302,7$ & $109.054,4$ & $\begin{array}{l}(1.590 \\
\text { en CyL) }\end{array}$ \\
\hline DIARIO DE BURGOS & - & $64.906,82$ & $33.117,7$ & $98.024,52$ & $\begin{array}{l}(8.096 \\
\text { en CyL) }\end{array}$ \\
\hline ABC NACIONAL & - & $35.846,25$ & $61.276,82$ & $97.123,07$ & 3.873 \\
\hline DIARIO PALENTINO & - & $61.024,66$ & $14.048,1$ & $75.072,76$ & $\begin{array}{l}(2.897 \\
\text { en CyL) }\end{array}$ \\
\hline LANZA & $32.277,02$ & $19.369,2$ & $19.733,89$ & $71.380,11$ & $5.800^{4}$ \\
\hline $\begin{array}{l}\text { EL SEMANAL DE LA } \\
\text { MANCHA }\end{array}$ & $14.133,53$ & $17.562,33$ & $13.320,88$ & $45.016,74$ & 1.541 \\
\hline TOTAL & $943.935,55$ & $2.188 .820,73$ & $1.880 .271,40$ & $5.013 .027,68$ & \\
\hline
\end{tabular}

Fuente: Elaboración propia a partir de los datos del Portal de Transparencia y OJD.. Con * aquellas publicaciones cuyo único dato disponible para C-LM corresponde a 2016.

\subsubsection{Radio y Televisión}

El sector de las emisoras de radio ocupa el segundo lugar en cuantía percibida, concentrando el $26,02 \%$ de los importes abonados en todo el periodo (4.257.374,23 euros), distribuidos en 53 emisoras. En este caso, se producen algunas diferencias fundamentadas en la fórmula de propiedad de las emisoras. Las tres grandes empresas, Ser, Onda Cero y Cope poseen emisoras propias y otras que son propiedad de empresas asociadas. Así, la publicidad emitida en las emisoras propiedad de los tres grupos se contrata de forma centralizada; en cambio, las emisoras asociadas son empresas independientes que figuran como contratistas del Acuerdo Marco.

En el sector radiofónico la variabilidad de las audiencias en el Estudio General de Medios con varias oleadas anuales dificulta tomar como indicador el número de oyentes. A la luz de los importes, se puede confirmar que la Ser ha percibido 1.779.851,41 euros, una cuantía notablemente superior a sus dos competidoras directas, Onda Cero (1.044.155,45 euros) y Cadena Cope (827.719,48 euros), si bien se justificaría con que esta emisora posee más estaciones comarcales. Si tomamos la emisora en su conjunto, es el segundo medio que concentra mayor volumen de inversión, después de las publicaciones de Promecal. En lo que se refiere a los importes, un dato llamativo es la asignación a la emisora local Ser Las Pedroñeras, cuyo ámbito de emisión es la comarca de la Mancha, con una cuantía percibida

\footnotetext{
11 El último dato disponible en OJD corresponde a 2011 (1.322 ejemplares); según la información del editor, la tirada actual está entorno a los 3.000 ejemplares.

12 Cifra de difusión del semanario aportada por sus responsables; la publicación no está auditada por OJD.
} 
superior a Ser Cuenca, emisora situada en la capital conquense. Ambas emisoras son empresas asociadas a la cadena y, por tanto, están en manos de empresarios locales.

Tabla 5. Ranking de emisoras de radio por importe recibido (importes superiores a 10.000 euros).

\begin{tabular}{|l|l|l|l|l|}
\hline \multicolumn{1}{|c|}{ RADIOS } & \multicolumn{1}{c|}{$\mathbf{2 0 1 6}$} & \multicolumn{1}{c|}{$\mathbf{2 0 1 7}$} & \multicolumn{1}{c|}{$\mathbf{2 0 1 8}$} & \multicolumn{1}{c|}{ Suma Total } \\
\hline SER CASTILLA-LA MANCHA & $330.911,54$ & $378.246,6$ & $438.226,51$ & $1.147 .384,65$ \\
\hline COPE CASTILLA-LA MANCHA & $191.198,44$ & $333.408,41$ & $303.112,63$ & $827.719,48$ \\
\hline $\begin{array}{l}\text { ONDA CERO CASTILLA-LA } \\
\text { MANCHA }\end{array}$ & $274.061,34$ & $303.530,68$ & $232.567,63$ & $810.159,65$ \\
\hline ONDA CERO LOCAL & - & $61.994,47$ & $172.001,33$ & $233.995,8$ \\
\hline $\begin{array}{l}\text { SER LAS PEDROÑERAS } \\
\text { RADIO AZUL }\end{array}$ & $46.275,91$ & $73.587,16$ & $74.516,94$ & $194.380,01$ \\
\hline SER CUENCA & $37.530,99$ & $73.698,31$ & $70.532,22$ & $181.761,52$ \\
\hline RADIO SURCO & $36.583,84$ & $53.710,68$ & $91.347,71$ & $181.642,23$ \\
\hline SER TOLEDO & - & $49.319,6$ & 43.560 & $92.879,6$ \\
\hline $\begin{array}{l}\text { SER CLM-EMISORAS CIUDAD } \\
\text { REAL }\end{array}$ & $37.955,28$ & $18.754,76$ & $9.981,05$ & $66.691,09$ \\
\hline CADENA 100 CLM & $30.535,36$ & $18.803,5$ & $2.620,95$ & $51.959,81$ \\
\hline SER MOTILLA RADIO 90 & $15.453,31$ & $19.991,74$ & $16.211,45$ & $51.656,5$ \\
\hline SER ALMADEN & $15.619,6$ & $14.846,53$ & $14.631,91$ & $45.098,04$ \\
\hline RADIO SANTAMARIA & $10.195,98$ & $12.174,54$ & $16.834,24$ & $39.204,76$ \\
\hline ESRADIO ALBACETE & $8.688,87$ & $11.617,9$ & $10.410,54$ & $30.717,31$ \\
\hline TOTAL & $1.035 .010,46$ & $1.423 .684,88$ & $1.496 .555,11$ & $3.955 .250,45$ \\
\hline
\end{tabular}

Fuente: Elaboración propia a partir de los datos del Portal de Transparencia.

En cuanto a la televisión, se puede reseñar que, en función del ámbito de cobertura, resulta paradójico que dos televisiones de ámbito local (Imás TV y Tele Hellín) estén por delante en cuantía percibida de La Regional TV, la única televisión de las cuatro que obtuvieron licencia autonómica de TDT que aún emitía en 2016 y $2017^{13}$. No obstante, hay que precisar que en esta cadena regional estaba integrada la televisión local TeleToledo, en funcionamiento aún, por lo que la empresa propietaria de ambas, Cadena Local TV, percibió 803.476,79 euros, más de un cuarto del importe global invertido en el sector.

13 En julio de 2017 cesó sus emisiones. 
Tabla 6. Ranking de empresas de televisión por importe

\begin{tabular}{|l|l|l|l|l|}
\hline \multicolumn{1}{|c|}{ TELEVISIÓN } & \multicolumn{1}{c|}{$\mathbf{2 0 1 6}$} & \multicolumn{1}{c|}{$\mathbf{2 0 1 7}$} & \multicolumn{1}{c|}{$\mathbf{2 0 1 8}$} & \multicolumn{1}{c|}{ Suma Total } \\
\hline IMAS TV & $167.645,55$ & $225.992,91$ & $212.126,31$ & $605.764,77$ \\
\hline TELETOLEDO & $74.464,37$ & $218.886,1$ & $228.760,18$ & $522.110,65$ \\
\hline $\begin{array}{l}\text { TELE HELLIN (GEINTEL } \\
\text { RADIO) }\end{array}$ & $136.717,4$ & $175.905,08$ & $100.137,67$ & $412.760,15$ \\
\hline TV LA REGIONAL & $240.528,64$ & $40.837,5$ & - & $281.366,14$ \\
\hline GUADA TV & & $20.908,8$ & $259.978,18$ & $280.886,98$ \\
\hline TV LA MANCHA & $87.464,85$ & $91.456,64$ & $82.036,31$ & $260.957,8$ \\
\hline $\begin{array}{l}\text { CANAL 4 MANCHA CENTRO } \\
\text { TV VILLARROBLEDO }\end{array}$ & $44.066,6$ & $64.350,8$ & $70.802,91$ & $179.220,31$ \\
\hline TELEVALDEPENAS & $27.188,7$ & $60.601,64$ & $67.924,56$ & $155.714,9$ \\
\hline CADENA LOCAL TV & & $36.717,45$ & $48.339,5$ & $85.056,95$ \\
\hline $\begin{array}{l}\text { CANAL DIOCESANO TV } \\
\text { TOLEDO }\end{array}$ & $24.526,7$ & $19.278,93$ & $22.459,42$ & $66.265,05$ \\
\hline TV ALMANSA & $7.511,68$ & $18.886,31$ & 17.787 & $44.184,99$ \\
\hline TELEAZUER & $19.176,56$ & $14.701,79$ & $8.160,24$ & $42.038,59$ \\
\hline ATRESMEDIA & $17.772,19$ & $17.515,96$ & - & $35.288,15$ \\
\hline TV GUADALAJARA (SOLARIS) & 9.680 & - & - & 9.680 \\
\hline ROBLEVISION-CANAL 4 & - & 3.872 & - & 3.872 \\
\hline TELERODA & 3.025 & - & - & 3.025 \\
\hline TOTAL & $859.768,24$ & $1.009 .911,91$ & $1.118 .512,28$ & $2.988 .192,43$ \\
\hline
\end{tabular}

Fuente: Elaboración propia a partir de los datos del Portal de Transparencia.

\section{Discusión y conclusiones}

A partir de los análisis efectuados y la revisión bibliográfica y jurídica, queda demostrado que la regulación es desigual en el territorio nacional, ya que todavía hay algunas comunidades que no han desarrollado legislación específica. La Ley 29/2005, Publicidad y Comunicación Institucional sólo afecta a las contrataciones de la AGE, por lo que no tiene que ser aplicada de forma obligatoria por las distintas administraciones autonómicas, aunque estas sí están sujetas a prohibiciones como las de llevar a cabo campañas de logros y objetivos de gestión; menoscabar con sus mensajes las políticas públicas de otras administraciones; incluir mensajes contrarios a los valores y principios constitucionales, o bien sean sexistas o discriminatorias, entre otras.

Se puede constatar que la normativa actual resulta insuficiente para garantizar la transparencia ya que la propia AGE y varias comunidades autónomas con legislación propia no hacen públicos sus datos (especificando inserciones por medio y cantidad 
facturada), lo que impide un control efectivo del gasto que supone la inversión en publicidad institucional.

En relación a la Junta de Comunidades de Castilla-La Mancha, los datos confirman que la implantación del Acuerdo Marco ha mejorado la transparencia en la distribución de las cuantías ya que, por primera vez, los datos de inversión son públicos. Otros aspectos positivos son el apoyo a la presencia de periodistas en los medios o el incremento de medios digitales sometidos a una auditoría (OJD Interactiva) que hace públicas sus mediciones. Ambas cuestiones indican un interés por favorecer medios profesionales, en detrimento de iniciativas particulares cuyos intereses no siempre están claramente identificados. Por otro lado, la fórmula facilita la competencia y concurrencia de todos los medios en igualdad de condiciones al no adjudicar directamente los planes de medios a agencias de publicidad, cuya gestión posterior puede ser más opaca. No obstante, hay margen de mejora en cuanto la publicación de los criterios de contratación ya que no es posible conocer qué indicadores se toman para valorar cuestiones como, por ejemplo, la calidad del contenido junto al que se publica el anuncio.

El estudio llevado a cabo en torno a la proporcionalidad entre las audiencias y el importe percibido por cada de una de las empresas de comunicación ha resultado efectivo para detectar que éste no es el único indicador a tener en cuenta, si bien no siempre se puede valorar en qué medida las asignaciones están justificadas. Aunque el desarrollo de una ley autonómica sería el siguiente paso, se puede concluir que la adopción de este sistema ha constituido un buen punto de partida para lograr un equilibrio y fomentar la cultura de la transparencia -tanto en empresas periodísticas como en la Administración - frente a la opacidad (y posible arbitrariedad) de Ejecutivos anteriores.

\section{Referencias bibliográficas}

Aguado, M. G.; Sanmartí, J.M.; Magallón, R. (2009). "España, Francia, Italia y Portugal: Cuatro modelos diferenciados de prensa euromediterránea", Observatorio (OBS) Journal, 9, pp. 62-84.

Alvarado, M. C.; De Andrés, S. (2005). "Una reflexión crítica sobre la publicidad estatal. El Estado ¿anunciante social?”. Telos: Cuadernos de comunicación e innovación, n. 64, pp. 67-73.

Alvárez-Peralta, M.; Franco, Y. G. (2018). “Independencia periodística y fondos públicos: la Comunicación Social Institucional como distorsión de la competencia en el mercado informativo". Historia y Comunicación Social, Vol. 23, n. 2. https://doi.org/10.5209/ HICS.62258

Benavides, J. (2010). "Qué pasa en España con las marcas y la publicidad en el ámbito de la comunicación institucional". Revista Icono14, Vol. 2, pp. 72-89. https://doi.org/10.7195/ ri14.v8i2.247

Blasco Gil, J. J. (2008). "Las ayudas públicas a la prensa de las Comunidades Autónomas españolas en 2007: tipología, cuantía de las subvenciones y sistemas de adjudicación". Telos: Cuadernos de comunicación e innovación, n.75.

Caro, A. (2008). "De la propaganda a la publicidad". Pensar la Publicidad, n. 2, pp. 9-12.

Colino, A. (2013). "Evolución de la financiación de los medios de comunicación en España. ¿Hacia un modelo más sostenible?”. Papeles de Europa. Instituto Complutense de Estu- 
dios Internacionales, vol. 26, n. 1, pp. 46-69. https://doi.org/10.5209/rev_PADE.2013. n26.42801

Cortés González, A. (2011). "La publicidad institucional en España. Una década en perspectiva". Razón y Palabra, Vol. 16, n. 75, pp. 1-23.

Curto, V. (2013). Escenarios de la publicidad institucional. Definición, contextualización, historia y operativa de la publicidad de las administraciones públicas. Tesis doctoral. Universitat Ramon Llull.

Edo, C. (1994). La crisis de la prensa diaria. La línea editorial y la trayectoria de los periódicos de Madrid. Barcelona: Ariel.

Escolar, A., 2014. "Publicidad institucional: aún pesebre y trabuco". Cuadernos de Periodistas, vol. 28, pp. 14-19.

Feliu Albaladejo, Á. (2009). Los ámbitos de la Publicidad Institucional. En Feliu, E. (coord.): Comunicación: memoria, historia y modelos. Madrid: Edipo, pp. 428-436.

Feliu García, E.; Feliu Albaladejo, M. Á. (2012). Publicidad institucional en la Comunidad Valenciana: la Generalitat como anunciante. En P. Peña Jiménez, M. Pacheco Rueda y E. Martínez Pastor (Coords.): Comunicación institucional y política. Madrid: Fragua.

Feliu García, E.; Feliu Albaladejo, M. Á. (2011). "La publicitat institucional a Catalunya". Comunicació. Revista de Recerca i d'Anàlisi. vol. 28, n. 2, pp. 47-67.

Feliu García, E.; Quintas, N. (2010). “La Xunta de Galicia como anunciante”. Comunicación presentada en el XII Foro de Investigación en Comunicación. La Comunicación institucional, (págs. 1-18). Madrid, 25-26 noviembre.

Fernández Alonso, I.; Blasco Gil, J. J. (2005). "Las ayudas públicas a la prensa en Cataluña (2000-2003)". Sphera Publica, n. 5.

Fernández Poyatos, M. D.; Feliu García, E. (2012). "La publicidad institucional de la Junta de Andalucía". Pensar la publicidad. Revista internacional de investigaciones publicitarias, vol. 6, n. 1, pp. 115-138. http://dx.doi.org/10.5209/rev_PEPU.2012.v6.n1.38659

Galletero Campos, B.; López Cepeda, A. M. (2018). “Ayudas directas y publicidad institucional a medios de comunicación en el escenario autonómico: indicadores y pautas de mejora". El profesional de la información, vol. 27, n. 3, pp. 682-691. https://doi. org/10.3145/epi.2018.may.21

Galletero Campos, B. (2018). Del periódico impreso al diario digital: Estudio de una transición en Castilla-La Mancha. Tesis doctoral: Universidad de Castilla-La Mancha.

Gaona Martínez, C.; Martínez Pastor, E. (2009). “Análisis de Género de las Campañas de Publicidad de La Dirección General de Tráfico”. Icono 14, vol. 7 n. 2, pp. 195-213. https:// doi.org/10.7195/ri14.v7i2.323

García López, M. (2001). Publicidad institucional: el Estado anunciante. Málaga: Universidad de Málaga.

García Uceda, M. (2001). Las claves de la publicidad. Madrid: Esic.

Guichot, E. (2016). "El reparto de reparto de la publicidad institucional entre los medios de comunicación; transparencia, objetividad y control". Revista Española de Derecho Administrativo, n. 175, pp. 85-125.

Martínez Pastor, E. (2016). "Estado de la cuestión de la publicidad institucional en España (2006-2012)". Poliantea, vol. 11, n. 21, pp. 105-128.

Martínez Pastor, E. (2012). "Publicidad Institucional de las Administraciones Públicas: marco jurídico y controversias". Comunicación y hombre, n. 8, pp. 51-63.

Martínez Pastor, E. y Vizcaíno-Laorga, R. (2008). "Publicidad institucional como fenómeno integrador ante la inmigración en España: régimen jurídico". Revista Latina de Comunicación Social, n. 63, pp. 90 - 98. 
Noelle Neumann, E. (1995). La espiral del silencio: opinión pública: nuestra piel social. Barcelona: Paidós.

Ortega Martínez, E. (1999). La comunicación publicitaria. Madrid: Pirámide.

Pineda, A. y Rey, J. (2009). "Propaganda y publicidad institucional: algunas consideraciones teóricas". Questiones publicitarias, n. 1, pp. 9-32. https://doi.org/10.5565/rev/qp.113

Psychogiopoulou, E. (2012). (Ed). Understanding Media Policies: A European Perspective. London, Reino Unido: Palgrave Macmillan.

Ríos Martín, D. (2016). Gestión de la publicidad institucional en los Ayuntamientos. El caso de los ayuntamientos de la Costa del Sol. Trabajo Fin de Máster. Universidad de Málaga.

Rodríguez López, M.; Robles Álvarez, D. (2016). "La publicidad institucional en España: Análisis de las campañas contra la violencia de género del gobierno (2006-2015)". Revista de Comunicación Vivat Academia, n. 134, pp. 83-104. http://dx.doi.org/10.15178/ va.2016.134.83-104

Sanmartí J.M.; Aguado, G.; Magallón, R. (2010). “Comparativa de modelos en las relaciones Prensa-Estado: Colombia, Italia, Francia, España y Portugal”. Palabra clave. https://doi. org/10.5294/pacla.2010.13.1.8

Segura García, R. (2015). "Evolución y efectividad de los spots de la DGT”. Opción, n. 31, pp. 1180-1200.

Vilches, F. (2010). La publicidad Institucional. En Robles, S. y Romero, V. (Coords.): Publicidad y lengua española. Un estudio por sectores, Sevilla - Zamora: Comunicación Social Ediciones y Publicaciones, pp. 190-202. 